G.M.H.J. Habets

Sterrewacht 'Sonnenborgh', Rijksuniversiteit Utrecht, Zonnenburg 2, 3512 NL Utrecht, The Netherlands

\title{
OBSERVATIONS AND EVOLUTIONARY SCENARIO
}

Rappaport \& Van den Heuvel (1982) suggested the following formation scenario for $B$ emission (Be)/X-ray binaries: the progenitor of the neutron star is the initial primary star with mass $M_{1} \simeq 10-20 M_{\theta}$, which during hydrogen-shell burning transfers mass to the companion (with $M_{2}<M_{1}$ ). The secondary is spun up due to disk-accretion (e.g. Packet 1981 ) and has become a rapidly rotating Be star $\left(M \simeq 10-20 M_{\odot}\right)$.

With this scenario the observed orbital perfods ( $P_{\text {orh }}^{\odot}$ ) $15 \mathrm{~d})$ of the Be/X-ray binaries can be explained. This was shown by Van den Heuvel (1983) by adopting an idealized period distribution for unevolved $0-$ and B-type close binaries with $P_{\text {orb }} \leqslant 30 \mathrm{~d}$ (see Fig. 1) and assuming conservative evolution, i.e. that mass and angular momentum are conserved during mass transfer. We have calculated the expected period distribution (epd) after mass transfer for $M_{1}=12 \quad M_{\theta}$ and $M_{1}=20 \quad M_{\theta}$ assuming non-conservative mass exchange and an initial, mean mass ratio $q_{0}$ of the components of 0.7 (Fig. 1). The period changes are calculated with the formalism of Vanbeveren et al. (1979) with their parameters $\alpha$ and $\beta$ chosen as 1 and 0.6 (i.e. $40 \%$ of the transferred mass leaves the

Fig. 1. The frequency distribution of orbital periods before and after mass exchange for several cases as explained in the text. The solid curve and the histogram represent the distribution of unevolved 0 - and $B$-type close binaries with periods $\leqslant 30 \mathrm{~d}$ after Van den Heuvel (1983).

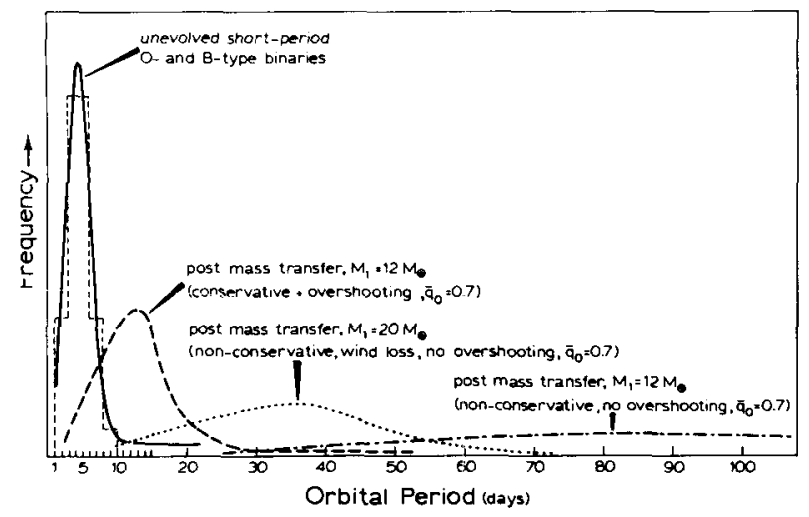


system with a small amount of angular momentum). In case of the $20 \mathrm{M}_{0}$ star stellar-wind mass loss before the mass transfer is included. The resulting epd of the $12 \mathrm{M}_{\Theta}$ and $20 \mathrm{M}_{\odot}$ star peaks at $86 \mathrm{~d}$ and $36 \mathrm{~d}$, res-

Fig. 2. The evolutionary scenario for the formation of highly eccentric Be/X-ray binaries from very massive close binaries by a symmetric supernova explosion. Between stages (1) and (2) and between (3) and (4) the massive star undergoes stellar-wind mass loss (the mean mass-loss rates are indicated). By spiral-in and loss of the common envelope a Wolf-Rayet star is formed. Later on, this star explodes and a neutron star is formed by core collapse. In stage (5) the outer layers of the secondary are spun up by strong tidal interaction at periastron. Hence, the equatorial velocity $v_{e}$ of the secondary increases and the secondary is suggested to become a Be star. An X-ray outburst may occur when the $\mathrm{Be}$ star overflows its 'Roche lobe' at periastron in stage (5). The scale of (3) is $\sim 20$ times that of (1) and (2). Bars indicate a distance of $10 \mathrm{R}$. The scenario may apply to the evolutionary history of A0538-66.

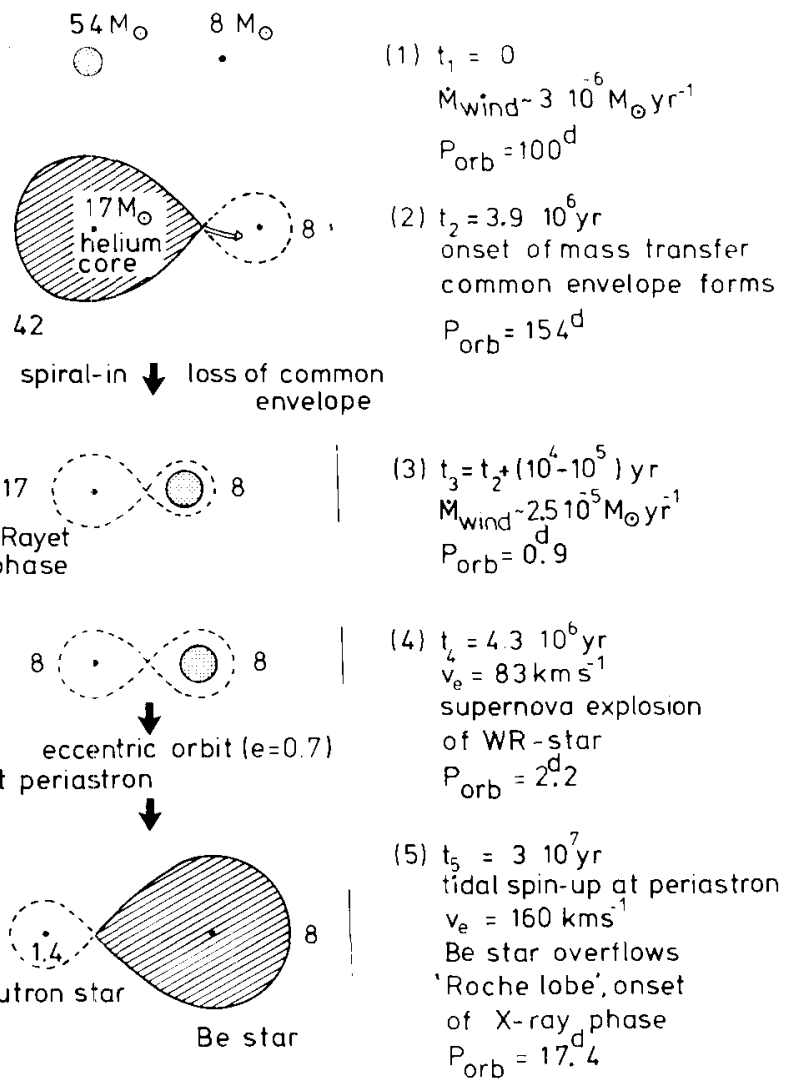


pectively, and is slightly different from the one in the conservative case. If convective overshooting occurs during core-hydrogen burning the mass-exchange remnant will be more massive (Doom 1984). As a result the epd's of the $12 \mathrm{M}_{\odot}$ and $20 \mathrm{M}_{\odot}$ stars which undergo overshooting, peak at $13 \mathrm{~d}$ and $10 \mathrm{~d}$. Finally, the periods increase by a factor $\leqslant 1.5-8$ (roughly) due to a possible second mass transfer from the initial primary to the secondary star during helium- or carbon-shell burning (cf. Habets $1986 \mathrm{a}, \mathrm{b})$, and by a factor $\leqslant 1.5\left(\leqslant 4\right.$, for $M_{1}=20 \mathrm{M}_{\odot}$ with overshooting) due to stellar-wind mass loss and the supernova (SN) explosion. Hence, these scenarios yield the observed range of $P$

For all above-mentioned scenarios holds that the final orbital eccentricity $e$ is small if the $\mathrm{SN}$ explosion is symmetric: the conservative and non-conservative cases yield $\mathrm{e}<0.1-0.2$; in case overshooting occurs, e may become $\sim 0.3$ for $M_{1}=20 \mathrm{M}_{0}$. Hence, these scenarios fail to explain the observed, large values of $e^{e}$ in a few $\mathrm{Be} / \mathrm{X}-$ ray binaries $(e \approx 0.3-0.8)$, cf. Hutchings et al. (1985). Furthermore, we do not readily obtain Be-star masses of $\sim 6-10 \mathrm{M}_{\odot}$, cf. Hutchings et al.

\section{ALTERNATIVE SCENARIOS}

Asymmetric SN explosions provide one way to yield the desired large values of $e$, but in this case $P_{\text {orb may increase as well as }}$ decrease. Here we suggest an alternative scenario in which highly eccentric Be/X-ray binaries are formed from very massive binaries in a symmetric SN explosion after highly non-conservative evolution as depicted in Fig. 2, cf. Van den Heuvel \& Habets (1984). Note that the resulting Be-star mass is $<10 \mathrm{M}_{\odot}$ in the case shown here.

Acknowledgements

The author thanks Prof. Dr. E.P.J. van den Heuve1 for stimulating discussions. This research was supported by the ZWO foundation for Astronomical Research in the Netherlands (project no. 782-371-024).

\section{References}

Doom, C. (1984). Astron. Astrophys., 138, 101.

Habets, G.M.H.J. (1986 a,b). Astron. Astrophys. (in press).

Hutchings, J.B., Crampton, D., Cowley, A.P., Olszweski, E., Thompson, I.B., \& Suntzeff, N. (1985). Publ. Astron. Soc. Pacific, 97, 418.

Packet, W. (1981). Astron. Astrophysics., 102, 17.

Rappaport, S. \& Van den Heuvel, E.P.J. (1982). In Be stars, eds. M. Jaschek \& H.-C. Groth, IAU Symp. 98, 327 .

Vanbeveren, D., De Grève, J.P., Van Desse1, E.L., \& De Loore, C. (1979). Astron. Astrophys., 73, 19.

Van den Heuve1, E.P.J. (1983). In Accretion-driven Stellar X-ray Sources, eds. W.H.G. Lewin \& E.P.J. van den Heuve1, pp. 303-341. Cambridge: Cambridge Univ. Press.

Van den Heuve1, E.P.J. \& Habets, G.M.H.J. (1984). Nature, $309,598$. 


\section{DISCUSSION FOLLOWING HABETS}

\section{Marlborough:}

Would you briefly explain the difference between a symmetric and an antisymmetric supernova explosion?

Habets:

The difference is that a symmetric explosion is suggested to tear apart the star in a completely symmetric way. However, in an anti- or asymmetric explosion the explosion is suggested to be not in the center, but off-center in the interior of the star. Thereby, a very large kick can be given to the system. Such a kick may produce large eccentricities, which may not be obtained by a symmetric explosion.

Granes:

In your opinion what would be the probable angular separation of the stars in term of $\mathrm{R}_{*}$ or other units? Do you think it can be observed interferometrically?

Habets:

In terms of solar radii, the initial orbit would have an orbital separation $a$ of 340 solar radii, i.e., say, 30 times $R_{*}$ of the 54 solar mass star. At the two final stages the orbital separation is at minimum $\sim 18$ solar radii, i.e., 2 to 4 times the stellar radius of the 8 solar mass star. Being non-expert, I would suggest that only the first two stages would be good cases to be studied interferometrically. However, the binary A0538-66 is in the LMC and, therefore, it is obviously too far away to be observed interferometrically in all stages of the evolutionary scenario here.

Collins:

Why does your scenario not show any accretion onto the secondary? You have lost over 50 solar masses from the system and none appears to have been accreted onto the secondary. This appears to be true even during the phase where the secondary is "spun-up". How is this done without mass exchange?

Habets:

The scenario has several stages where mass accretion can occur. However, in cases of stellar-wind mass loss and in cases of Roche lobe overflow, or common envelope evolution, the timescales of mass accretion to the secondary are much shorter than the thermal KelvinHelmholtz timescale of the secondary. This would not allow much mass-accretion. As to the common- envelope stage, I would suggest that it is very similar to the case studied by Meyer and Meyer-Hofmeister in 1979. So, I refer you to that paper for more details. As to the effects of the supernova explosion, I refer to my paper (1986a) where it is argued that impact effects are small. The secondary is spun up by tidal interaction before and after the SN explosion. Of course, a few percent of the mass accreted would be sufficient to spin up the secondary, c.f. Packet (1981).

Underhill:

One phase of your model primary is called a "Wolf-Rayet phase". That is a speculative misnomer. The type "Wolf-Rayet" is assigned only on the basis of particular sets of emission lines in the spectrum. You have not shown that your model will provide such a spectrum. My colleague A.K. Bhater and I (Bhater and Underhill 1986) have shown that particular conditions are needed in the atmosphere to produce a "WR" spectrum. The conditions include normal $\mathrm{H} / \mathrm{He}$ composition. I object strongly to the nomenclature you have used; it is fundamentally misleading. I note you do not assign spectral types to 
other stages in the evolution of your massive models. That caution should be exercised in your full study. Identifying a model star with a real type of star requires detailed study of conditions in the atmosphere of the star.

van den Heuvel:

I propose to call the 17 solar mass star a helium star instead of a Wolf-Rayet star. 